\title{
Anatomical and biomechanical traits of broiler chickens across ontogeny. Part I. Anatomy of the musculoskeletal respiratory apparatus and changes in organ size.
}

Genetic selection for improved meat yields, digestive efficiency and growth rates have transformed the biology of broiler chickens. Modern birds undergo a 50-fold multiplication in body mass in just six weeks, from hatching to slaughter weight. However, this selection for rapid growth and improvements in broiler productivity is also widely thought to be associated with increased welfare problems as many birds suffer from leg, circulatory and respiratory diseases. To understand growth-related changes in musculoskeletal and organ morphology and respiratory skeletal development over the standard six-week rearing period, we present data from post-hatch cadaveric commercial broiler chickens aged 0, 2, 4 and 6 weeks. The heart, lungs and intestines decreased in size for hatch to slaughter weight when considered as a proportion of body mass. Proportional liver size increased in the two weeks after hatch but decreased between 2 and 6 weeks. Breast muscle mass on the other hand displayed strong positive allometry, increasing in mass faster than the increase in body mass.

Contrastingly, less rapid isometric growth was found in the external oblique muscle, a major respiratory muscle that moves the sternum dorsally during expiration. Considered together with the relatively slow ossification of elements of the respiratory skeleton, it seems that rapid growth of the breast muscles might compromise the efficacy of the respiratory apparatus. Furthermore, the relative reduction in size of the major organs indicates that selective breeding in meat-producing birds has unintended consequences that may bias these birds toward compromised welfare and could limit further improvements in meat-production and feed efficiency. 
1 Peter G. Tickle and Jonathan R. Codd

2 Faculty of Life Sciences, University of Manchester, Manchester, UK

3 Heather Paxton, Jeffery W. Rankin and John R. Hutchinson

4 Structure \& Motion Laboratory, Department of Comparative Biomedical Sciences, The Royal

5 Veterinary College, University of London, Hatfield, Hertfordshire, UK 
$7 \quad$ Genetic selection for improved meat yields, digestive efficiency and growth rates have 8 transformed the biology of broiler chickens. Modern birds undergo a 50-fold multiplication in

9 body mass in just six weeks, from hatching to slaughter weight. However, this selection for rapid 10 growth and improvements in broiler productivity is also widely thought to be associated with 11 increased welfare problems as many birds suffer from leg, circulatory and respiratory diseases. To 12 understand growth-related changes in musculoskeletal and organ morphology and respiratory 13 skeletal development over the standard six-week rearing period, we present data from post-hatch 14 cadaveric commercial broiler chickens aged 0,2, 4 and 6 weeks. The heart, lungs and intestines 15 decreased in size from hatch to slaughter weight when considered as a proportion of body mass. 16 Proportional liver size increased in the two weeks after hatch but decreased between 2 and 6 17 weeks. Breast muscle mass on the other hand displayed strong positive allometry, increasing in 18 mass faster than the increase in body mass. Contrastingly, less rapid isometric growth was found 19 in the external oblique muscle, a major respiratory muscle that moves the sternum dorsally during expiration. Considered together with the relatively slow ossification of elements of the respiratory skeleton, it seems that rapid growth of the breast muscles might compromise the efficacy of the respiratory apparatus. Furthermore, the relative reduction in size of the major organs indicates

23 that selective breeding in meat-producing birds has unintended consequences that may bias these

24 birds toward compromised welfare and could limit further improvements in meat-production and 25 feed efficiency.

\section{INTRODUCTION}

Genetic selection in domesticated broiler chickens has brought about significant improvements in the form of increasing meat yields and growth performance. Growth rates in intensively reared industrial broiler chickens have consistently accelerated such that a $300 \%$ increase has been engineered in the past 60 years, from $25 \mathrm{~g}$ per day in the $1950 \mathrm{~s}$ to $100 \mathrm{~g}$ per day in the modern bird (Knowles, et al., 2008). Consequently the optimal slaughter mass of

32 approximately $3 \mathrm{~kg}$ is reached in six rather than 16 weeks (Griffin and Goddard, 1994;

33 Havenstein, et al., 1994a; Govaerts, et al., 2000). Maximising pectoral (breast) muscle mass is a 34 primary target for selection. Compared to ancestral varieties, pectoral hypertrophy in the broiler 35 chicken has resulted in an approximate doubling in muscle size, making up $20 \%$ of total body 
mass in the modern bird (Havenstein, et al., 2003b; Schmidt, et al., 2009). Mounting evidence suggests that selection for such economically desirable traits in the modern broiler has been accompanied by reduced welfare (Julian, 1998; Knowles, et al., 2008) and increased mortality (Havenstein, et al., 1994a; Havenstein, et al., 2003a). Considerable research is being directed toward understanding welfare problems such as the multitude of leg pathologies that may affect locomotion in broiler chickens (Kestin, et al., 1992; Bradshaw, et al., 2002; Corr, et al., 2003a; Corr, et al., 2003b; Knowles, et al., 2008; Paxton, et al., 2010), cardiac (Wilson, et al., 1988) and pectoral (Randall, 1982) myopathies, pulmonary hypertension (Wideman, 2001) and ascites (Wilson, et al., 1988; Julian, 1993). The prevalence of these conditions indicates that further improvements in industry-efficiencies and meat production may be constrained by the physiological capabilities of broilers because skeletal, cardiac, respiratory and digestive systems appear to be close to their functional limit.

Characterising the relationships between body mass and organ, skeleton and muscle size are crucial to our understanding of animal physiology. Physical scaling rules determine the structural and functional consequences of changes in size and therefore exert a profound effect on organismal form (Schmidt-Nielsen, 1984). Understanding the relative growth and size of organs and muscles is important in broilers as it can help to better understand the diseases that they suffer from. For example, the broiler heart and brain become progressively smaller as a proportion of body mass over development, unlike in ancestral breeds (Jackson and Diamond, 1996; Schmidt, et al., 2009). In contrast, selection for faster growth and muscle mass is reflected in the proportionally greater intestine mass and accelerated pectoral growth in broiler chickens (Jackson and Diamond, 1996; Konarzewski, et al., 2000; Schmidt, et al., 2009). These relationships demonstrate how artificial selection in the broiler has resulted in developmental trade-offs; reallocation of resources to maximize nutrient absorption and pectoral muscle mass has coincided with a relative decrease in the size of other organs (Havenstein, et al., 1994b; Jackson and Diamond, 1996; Havenstein, et al., 2003b; Schmidt, et al., 2009). To better understand the effects of intensive selection on broilers, more information is required on how increasing body mass and growth rate has shaped their anatomy and physiology across ontogeny. Therefore, in this paper we present data on how organ and muscle growth varies with increasing body mass in a commercial broiler strain.

Broiler chickens suffer from respiratory problems that may be related to their rapid musculoskeletal development ( $\underline{\text { Julian, 1998) }}$ ) and their potential to outgrow pulmonary capacity (Wideman, 2001). The avian respiratory system can be considered as a two-part mechanism 
69 comprising a pump (musculoskeletal) and gas exchanger (lung). The primary ventilatory 70 mechanism in birds consists of dorsal and ventral movements of the ribs and sternum (Zimmer, 71 1935; Claessens, 2009) that affect air sac volume, thereby facilitating a unidirectional flow of air 72 through the lung (Bretz and Schmidt-Nielsen, 1971; Scheid and Piiper, 1971). Uncinate 73 processes, which are small bones that extend caudodorsally from the vertebral ribs, have been 74 shown to function as levers that assist rib and sternal movements during breathing (Tickle, et al., 75 2007). Respiratory movements of the skeleton generate pressure changes within the thorax that 76 are necessary to drive inhalation and exhalation, both of which are active processes, driven by

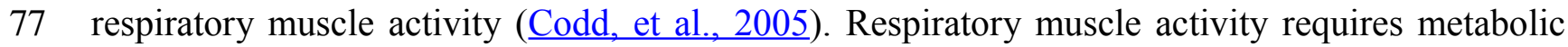
78 energy consumption. However, studies in guinea fowl (Numida meleagris) (Markley and Carrier, 79 2010) have demonstrated that breathing constitutes only $2 \%$ of whole-organism metabolism (Markley and Carrier, 2010). In contrast, research on load carrying (Tickle, et al., 2010) and behavioural energetics (Tickle, et al., 2012) indicate a higher cost of breathing in barnacle geese. In particular, carrying extra weight on the sternum, analogous to increased pectoral muscle mass seen in broiler chickens, is energetically expensive compared to an equivalent weight carried on the back (Tickle, et al., 2010). Furthermore, barnacle geese have been shown to achieve metabolic savings by changing posture; when compared to sitting, standing is associated with a $25 \%$ higher resting metabolic rate (Tickle, et al., 2012). The higher cost of standing has been in part attributed to the energetic cost of moving the heavy weight of the sternum with each breath, which does not occur while sitting (Tickle, et al., 2012). Understanding movements of the sternum are important when considering how selection has shaped morphology in domestic birds due to the selection for enhanced pectoral growth in meat-producing domestic ducks (Gille and Salomon, 1998; Maruyama, et al., 1999), turkeys (Swatland, 1979) and broiler chickens

92 (Havenstein, et al., 1994b; Govaerts, et al., 2000; Havenstein, et al., 2003b; Schmidt, et al., $93 \underline{2009})$. respiratory skeleton. In the domestic turkey (Meleagris gallopavo) a trend for delayed ossification, especially in the uncinate processes, was identified that potentially constrained respiratory performance due to the decreased rigidity of cartilaginous compared to fully ossified

98 bones; cartilaginous uncinate processes will yield under muscle strain before comparable ossified 99 bone would, making them less effective levers (Tickle and Codd, 2009). Furthermore, the growth 100 trajectory of respiratory muscles in the broiler is unknown. Scaling of respiratory muscle growth 101 in proportion to overall body size and, perhaps most importantly when we consider the dorso- 
102 ventral breathing movements of the sternum, the pectoral muscles is likely a key factor in

103 determining how effectively and efficiently breathing functions in broiler chickens. To better

104 understand the respiratory problems apparent in broiler chickens, an analysis of how the skeleton 105 develops is necessary. Here we present a description of developmental changes in the 106 musculoskeletal elements of the broiler chicken respiratory system together with an evaluation of 107 how organ size scales with increasing body mass.

\section{MATERIALS AND METHODS}

\section{Specimens}

110 Broiler chickens from a popular commercial strain were obtained from a commercial supplier. 111 Birds were sampled in a post-hatch growth series between days 1 and 42 (i.e. weeks 0 to 6), 112 corresponding to a 50x range in total body mass $\left(\boldsymbol{M}_{\mathbf{b}}\right)$ (Table 1).

\section{Musculoskeletal growth}

$114 M_{\mathrm{b}}$, pectoralis major (pectoralis), pectoralis minor (supracoracoideus) and external 115 oblique muscle mass $\left(\boldsymbol{M}_{\mathbf{m}}\right)$, wing and ribcage mass, fibre length $\left(\boldsymbol{L}_{\mathbf{f}}\right)$ and pennation angle $(\boldsymbol{\theta})$ were 116 measured using an electronic balance $( \pm 0.001 \mathrm{~g})$, ruler $( \pm 1 \mathrm{~mm})$ and protractor $\left( \pm 1^{\circ}\right)$. To account 117 for variation within muscle architecture, $L_{\mathrm{f}}$ and $\theta$ were calculated as the mean of five 118 measurements in each muscle. Physiological cross-sectional area (PCSA) was calculated for each 119 muscle (Eq. 1; Sacks and Roy, 1982):

$$
\mathrm{PCSA}=M_{\mathrm{m}} * \cos \theta / \rho * L_{\mathrm{f}}
$$

121 Density of muscle tissue $(\rho)$ was assumed to be $1.06 \mathrm{~g} \mathrm{~cm}^{-3}$ (Mendez and Keys, 1960; Paxton et al, 122 2010). Linear measurements of the sternum and uncinate processes were recorded using a ruler $123( \pm 1 \mathrm{~mm})$. Average length of the uncinate processes was calculated from those occurring on ribs 21245 since these processes were found in all specimens. Girth was measured around the 125 circumference of the thorax, tucked under the wings. One-way ANOVA was used to test for 126 differences in morphology, using mean values of specimens from each developmental stage.

127 Justification for using parametric analysis was based upon the results of a Shapiro-Wilk test and 128 the normal quantile-quantile plot that assessed assumptions of data normality (results displayed in 129 Appendix). 
130 The scaling relationships between musculoskeletal characters and body mass were 131 determined using reduced major axis (RMA) linear regression, a method that is suitable since it 132 takes into account variation in both $\mathrm{x}$ and $\mathrm{y}$ axes (Rayner, 1985; Sokal and Rohlf, 1995). All 133 regression analyses were performed on $\log 10$-transformed data to establish allometric equations 134 in the form:

135

$$
\log y=\log a+b \log x
$$

137

where $a$ represents the intercept and the exponent $b$ represents the slope of the line equation. Upper and lower 95\% confidence intervals (CI) and the $\mathrm{R}^{2}$ value were calculated for each regression line slope. RMA analyses were performed in the PAST statistical program (Hammer, et al., 2001). Assuming geometric similarity (i.e., isometry) over ontogeny, all dimensions are expected to scale in proportion to each other meaning that lengths should scale to $M_{\mathrm{b}}{ }^{0.33}$, areas to $M_{\mathrm{b}}{ }^{0.67}$ and masses to $M_{\mathrm{b}}{ }^{1.00}$. Isometric scaling was assumed where the regression slope $\pm 95 \% \mathrm{CI}$ overlapped the expected value.

\section{Histological staining}

The ossification pattern of the thoracic skeleton over ontogeny was examined using the histochemical staining protocol of Tickle and Codd (2009). All muscle tissue was removed using dissection, the preparation cleaned by immersion in a $1 \%$ potassium hydroxide $(\mathrm{KOH})$ solution and then the skeleton was treated with solutions of alcian blue (uptake corresponds to cartilage) and alizarin red (uptake corresponds to bone). Photographs of stained specimens were taken using a light microscope (Leica MZ9s; Leica Microsystems, Milton Keynes, UK) and subsequently analysed in Leica image analysis software. For comparison of structural properties, relative area of bone and cartilage was calculated for the uncinate process that projects from the fourth vertebral rib in all specimens (Tickle and Codd, 2009).

\section{Organ Development}

Heart and lung mass follow a negative allometric growth pattern, decreasing relative to body mass over the six-week growth period (Tables 1 and 2; Fig. 1A). Heart mass decreases from $0.74 \%$ to $0.53 \%$ of body mass over the growth period, while proportional lung mass reduces by 
159 almost half, decreasing from $1.04 \%$ to $0.55 \%$ (Tables 1 and 2; Fig. 1B). Proportional liver mass

160 significantly increased between 0 and 2 weeks, reached a peak of $3.63 \%$ on day 14 , then

161 decreased between 2 and 6 weeks when it accounted for 2.48\% of $M_{\mathrm{b}}$ (Table 1; Fig. 1C). Taking 162 all data into account indicated that overall liver mass followed an isometric growth pattern; i.e., 163 in direct proportion to increasing $M_{\mathrm{b}}$ (Table 2 ). Repeating the scaling analysis for birds only aged 164 between 0 and 2 weeks found positive allometric growth, $M_{\mathrm{b}}{ }^{1.10}$ while birds aged between 2 and 6 165 weeks had negative allometric growth, $M_{\mathrm{b}}{ }^{0.76}$ (Table 2; Fig. 1C). Total intestine mass was found to 166 strongly decline as a proportion of $M_{\mathrm{b}}$ over growth with a negative allometric regression slope of $167 M_{\mathrm{b}}^{0.75}$ (Tables 1 and 2; Fig. 1D).

\section{Carcass Parts}

169 No significant difference was detected between proportional wing masses with increasing age, 170 indicating a directly proportional relationship with $M_{\mathrm{b}}$. After accounting for variation due to body mass, girth significantly increased during growth (Table 3), reflecting a relative lateral expansion

172 of the thorax. In contrast, as a proportion of $M_{\mathrm{b}}$, ribcage mass was significantly lower at day 28 than at days 14 or 42 (Table 3) whereas normalised keel length displayed a trend for increased length, being highest in 42-day birds (Table 3).

175

176

177

178

179

180

181

182

183

184

185

186

187

188

\section{Thoracic Anatomy}

Pectoralis major and minor (i.e., M. pectoralis and M. supracoracoideus) $M_{\mathrm{m}}$ increased as a proportion of $M_{\mathrm{b}}$ over development, showing strong positively allometric growth (Table 4 and 5). The growth of these muscles was defined by two phases: an initial rapid increase in $M_{\mathrm{m}}$ between weeks 0 and 2 was followed by a relatively slower increase between weeks 2 and 6 (Table 5; Fig. 2). Sternal keel length and depth developed with positive allometry, increasing above the expected geometric scaling exponent $\left(M_{\mathrm{b}}{ }^{0.33}\right)$ (Table 6), while mean uncinate process length scaled to $M_{\mathrm{b}}{ }^{0.30}$, indicating reduced length with increasing body mass. Growth of external oblique muscle was found to increase in direct proportion to increasing body mass (Table 6).

\section{Skeletal Development}

Ossification of the uncinate processes commences at around the time of hatch; 15-day embryos have entirely chondrified processes whereas one-day old chicks show 40\% ossification (Table 7; Fig. 3). Uncinate process bone synthesis proceeds from the midpoint in proximal and distal directions and overall bone area plateaus at $\sim 77 \%$ of total uncinate process area at 40 days old. 
189 The remaining 23\% remains cartilaginous, shared between tip and base (Table 7). At hatch the

190 sternum exhibits ossification in the most proximal portion in addition to centres of ossification in

191 the caudolateral processes. While bone growth extends distally along the sternum, at slaughter 192 age ossification of the sternal keel remains incomplete (Fig. 3)

\section{DISCUSSION}

194

195

196

197

198

199

200

201

202

203

204

205

206

207

208

209

210

211

212

213

214

215

216

217

218

\section{Organ Development}

Our observations confirm a decrease in heart and lung mass relative to body mass over development (Tables 2 and 3). These findings mirror the reduction in relative heart (Havenstein, et al., 1994b; Govaerts, et al., 2000; Thaxton, 2002; Havenstein, et al., 2003b; Schmidt, et al., 2009) and lung mass (Havenstein, et al., 1994b; Govaerts, et al., 2000; Havenstein, et al., 2003b) seen over ontogeny in Ross-type broiler breeds. While absolute mass of the heart is higher than found in unselected lines, proportional mass is lower at hatching and the difference progressively increases over development (Schmidt, et al., 2009). Similarly, lung mass progressively declines as a proportion of body mass over development in our broiler strain, mirroring the negative allometric lung growth in Ross broilers $\left(M_{\mathrm{b}}{ }^{0.84}\right.$ compared to $M_{\mathrm{b}}{ }^{0.86}$ in this study) (Govaerts, et al., 2000). Considering our results together with previous reports indicates that reduced circulatory and respiratory capacity is an unintended consequence of genetic selection for rapid growth and high $M_{\mathrm{b}}$. The reduction in broiler heart and lung mass compared to slower-growing, lighter breeds can be considered to be a contributing factor in the increased mortality and disease in modern broilers (Wideman, 2001; Havenstein, et al., 2003b). Further developments in genetic selection for increasing growth and improving breast yields may be constrained by limited respiratory and circulatory functional capacity. The increased incidence of physiological problems and mortality (Havenstein, et al., 1994a; Havenstein, et al., 2003a) in modern broilers indicates that these systems are already working close to maximal levels to satisfy the physiological demands of growth.

A complex pattern of liver growth was revealed, with organ mass growing proportionally faster than overall body mass growth in the two weeks after hatch, followed by a proportional decrease in mass between two weeks and six weeks post-hatch. Liver development in Ross-type broilers follows a similar pattern of proportionally decreasing after a period of rapid growth soon

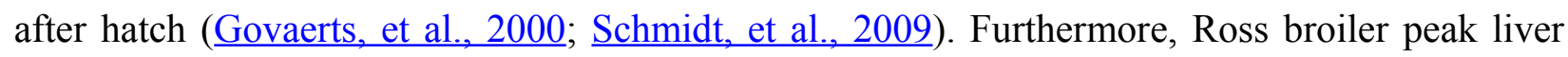


mass as a proportion of $M_{\mathrm{b}}$ was reached on day 7 (3.80\%) ( $\underline{\text { Schmidt, et al., 2009) }}$ ) and day 8

220 (3.38\%) (Govaerts, et al., 2000), although the findings presented here do not include birds 221 sampled at these stages. Nevertheless, proportional liver mass at day 14 is similar between our

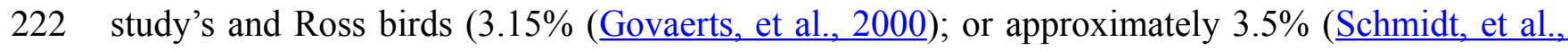
223 2009) of $M_{\mathrm{b}}$. The relatively fast pace of growth in the first two weeks, followed by proportional 224 decline, indicates that the liver matures rapidly, ready for the transition from fat-rich yolk stores

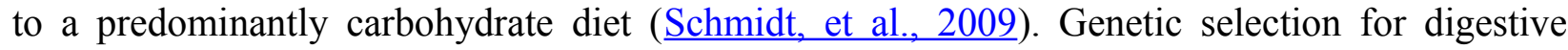
efficiency in broilers may directly influence the developmental profile of the liver due to the important function it plays in carbohydrate and fat metabolism.

Total intestine mass declined relative to $M_{\mathrm{b}}$ during growth, which is similar to previous reports of proportional decline in intestine mass over development in Ross-type broilers (Iji, et al., 2001; Schmidt, et al., 2009). Absolute and proportional values of the intestine are higher in this study, potentially in part due to a larger intestine in this commercial broiler strain and also in part due to methodological differences; here we report intestine mass and contents rather than the empty intestine mass (Iji, et al., 2001). Exhaustion of the yolk sac contents presumably accounts for the large $(6.9 \%)$ decline in proportional intestine mass during the first 14 days of post hatch development (Viera and Moran, 1999). After this period, relative intestine mass decreases by approximately $2-2.5 \%$ every 14 days (Table 1), which is similar to previous reports (calculations using the data provided in table 2 of Iji et al. (2001) and figure 5 of Schmidt et al (2009) indicate an approximate decrease of $2.4 \%$ ). Schmidt et al (2009) reported complicated post-hatch intestinal growth, with positive allometric growth in the first seven days followed by a sustained period of negative allometric growth, possibly reflecting an early maturation of the digestive system (Govaerts, et al., 2000). To balance this relative mass loss and maintain high feed

242 efficiency, morphometric changes occur during this period to increase surface area for absorption 243 of nutrients from ingested food (Schmidt, et al., 2009).

\section{Carcass Parts}

While proportional wing mass did not significantly change over growth the overall average yield of $7.0 \%$ is below that reported in Ross broilers (8.6\%: Havenstein, et al, 1994b; 7.9\%: Havenstein, et al, 2003b). The absence of significant change in wing mass is consistent

248 with the absence of flight as a locomotor mode and the reliance on leg muscles for terrestrial 249 locomotion, and occurs despite steep increases in pectoral muscle mass. Girth in 42-day-old 250 broilers was very similar to a recent report for pureline and broiler birds (Paxton, et al., 2013). 
251 Presumably the rapid increase in pectoral muscle mass is a factor in the reduced proportional 252 ribcage mass over development. Normalised sternal keel length was found to increase over 253 growth, indicating that the area available for pectoral muscle attachment is greater as the birds 254 reach slaughter weight, consistent with the rapid proportional increase in pectoral mass.

\section{Thoracic Anatomy}

The relationship between increasing pectoral mass and body mass was positively allometric, indicating that the breast muscles became proportionally much larger over the sixweek growth period. Allometric relationships were stronger than those reported for Ross broilers (Govaerts, et al., 2000; Schmidt, et al., 2009), indicating that the birds in this study were able to lay down breast muscle more rapidly during development. Overall breast muscle size as a proportion of $M_{\mathrm{b}}$ was similar to the values reported for Ross (Govaerts, et al., 2000; Havenstein,

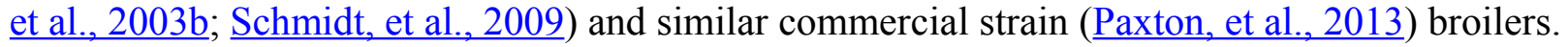

Allometric scaling was found for sternal keel dimensions; this proportional increase in the size of the sternum over development presumably enables fast growth of the breast muscles. In contrast, average uncinate process length was found to decrease over growth, scaling with a slight negative allometry. Uncinate processes act as levers for movement of the ribs and sternum during breathing, and overall length is an important factor that determines the magnitude of this leverage (Tickle, et al., 2007). Therefore, the relative shortening of uncinate processes might correspond to a decrease in ventilatory performance during growth. allometry during growth it was expected that the external oblique muscle would undergo similar growth to maintain functional efficacy. This muscle attaches to the base of uncinate processes and the sternal margin and is active during the exhalation phase of breathing, when it elevates the sternum dorsally (Codd, et al, 2005). Our data indicate however that the external oblique muscle becomes progressively smaller in comparison to the breast muscles as the birds age, and may be

276 less able to maintain adequate expiration. Further evidence is provided by the isometric growth of

$277 L_{\mathrm{f}}$ and PCSA, since maximum force-generating capacity increases in direct proportion to muscle

278 area (Lieber and Friden, 2000). To our knowledge, no comparable data on respiratory muscle 279 development exist, so it is difficult to establish whether this pattern of growth is unique to the 280 broiler or more widespread among ancestral fowl breeds and other species. Coupled with the 281 decreased proportional heart and lung masses, however, selection for meat-producing traits 282 appears to have the undesirable effect of compromising the engine of aerobic metabolism; i.e., 
283 the respiratory system. Clearly more research into the respiratory anatomy in birds is required to

284 understand the potential trade-offs between breast growth and breathing performance.

\section{Skeletal Development}

286 The broiler skeleton ossifies following a pattern similar to other domestic poultry (

287 1980; Maxwell, 2008; Tickle and Codd, 2009). Ossification of skeletal characters, however, is 288 seen to develop at different time points. For example, the long bones of the leg and wing are 289 almost entirely ossified at hatch, while the sternum, tip of the scapula and proximal and distal 290 portions of the ribs ossify after hatch (Fig. 3). Ossification of uncinate processes begins around 291 the time of hatch, in agreement with an earlier report in the chicken (Hogg, 1980), when bone 292 replacement of cartilage becomes apparent in the midpoint of the shaft (Table 7; Fig. 3). Rapid 293 synthesis of bone tissue must occur in the days immediately prior to hatch, considering that the processes are entirely chondrified six days before hatch (Table 7). Onset of ossification therefore coincides with the initial in ovo respiratory skeleton movements and transition from chorioallontoic gas exchange to pulmonary ventilation (Menna and Mortola, 2002); perhaps this mechanical stimulus prompts remodeling of uncinate process structure toward ossified tissue. Uncinate process growth in the broiler is similar to development in the domestic turkey (Tickle and Codd, 2009) in that ossification remains incomplete, with a plateau in bone growth at around $80 \%$ of total process area. An interesting difference between domestic turkey and chicken is the lack of a bony symphysis between the base of the process and adjoining rib in the broiler (Tickle and Codd, 2009). A cartilaginous base may have significant implications for the functional efficacy of the uncinate process because they act as force-amplyfying levers for muscle contractions during rib movement (Tickle, et al., 2007). The cartilaginous portion of uncinate processes has a lower elastic modulus, or is less stiff, than the ossified area which might compromise the lever function of the uncinate processes (Tickle and Codd, 2009). It is possible that an ossified connection between uncinate process and rib would form later in development, as is seen in the domestic turkey between days 64 and 94 post-hatch (Tickle and Codd, 2009), suggesting that immaturity of the skeleton even at slaughter weight may have a functional impact on breathing in the broiler.

Ossification of the sternum is incomplete over the six-week period, with specifically the 312 distal portion of the keel remaining chondrified. The cartilaginous distal portion of the sternum is 313 common to meat-producing and laying strains (Breugelmans, et al., 2007) and presumably 314 reflects the reduced or negligible importance of flight as a means of locomotion in domestic fowl; 
315 without need for a sturdy anchor to accommodate and dissipate the strong contractions of

316 pectoral muscles during flapping flight, it seems the energetic resources are directed to growing

317 muscle tissue rather than synthesis of bone for the sternum.

318 The rapid development of body mass in broilers is not mirrored by accelerated 319 ossification of the respiratory skeleton. Instead the skeleton appears to develop in a pattern

320 similar to other poultry, indicating that this process is tightly conserved within galliform birds.

321 Given the potential compromise in respiratory performance due to cartilaginous skeletal elements

322 coupled with a large sternal weight, selection for faster and increased ossification may provide a

323 benefit to broiler health.

$324 \quad$ Here we have considered the how the growth trajectories of major organs and respiratory 325 elements are affected by increasing body mass in broilers. In contrast to the strong pectoral 326 growth, major organs become proportionally smaller with age and development of the respiratory 327 pump is delayed. Our findings indicate that selection for rapid growth and large breast muscles 328 could have the unintended consequence of negatively affecting broiler physiology by 329 compromising the function capacity of cardiovascular and respiratory systems.

330

\section{ACKNOWLEDGMENTS}

331 We are thankful to Cobb-Vantress Inc. for providing broiler chicken specimens.

\section{REFERENCES}

333 Bradshaw, R. H., R. D. Kirkden, and D. M. Broom. 2002. A review of the aetiology and 334 pathology of leg weakness in broilers in relation to welfare. Avian and Poultry Biology Reviews 335 13:45-103. doi 10.3184/147020602783698421

336 Bretz, W. L., and K. Schmidt-Nielsen. 1971. Bird respiration: flow patterns in the duck lung. 337 Journal of Experimental Biology 54:103-118.

338 Breugelmans, S., S. Muylle, P. Cornillie, J. Saunders, and P. Simoens. 2007. Age determination of 339 poultry: a challenge for customs. Vlaams Diergeneeskundig Tijdschrift 76:423-430.

340 Claessens, L. P. a. M. 2009. The skeletal kinematics of lung ventilation in three basal bird taxa 341 (emu, tinamou, and guinea fowl). Journal of Experimental Zoology Part a-Ecological Genetics 342 and Physiology 311A:586-599. doi 10.1002/Jez.501 
343 Codd, J. R., D. F. Boggs, S. F. Perry, and D. R. Carrier. 2005. Activity of three muscles associated 344 with the uncinate processes of the giant Canada goose Branta canadensis maximus. Journal of 345 Experimental Biology 208:849-857. doi 10.1242/jeb.01489

346 Corr, S. A., M. J. Gentle, C. C. Mccorquodale, and D. Bennett. 2003a. The effect of morphology 347 on the musculoskeletal system of the modern broiler. Animal Welfare 12:145-157.

348 Corr, S. A., M. J. Gentle, C. C. Mccorquodale, and D. Bennett. 2003b. The effect of morphology 349 on walking ability in the modern broiler: A gait analysis study. Animal Welfare 12:159-171.

350 Gille, U., and F. V. Salomon. 1998. Muscle growth in wild and domestic ducks. British Poultry 351 Science 39:500-505.

352

353

354

355

356

357

358

359

360

361

362

363

364

365

366

367

368

369

370

371

372

373
Govaerts, T., G. Room, J. Buyse, M. Lippens, G. De Groote, and E. Decuypere. 2000. Early and temporary quantitative food restriction of broiler chickens. 2. Effects on allometric growth and growth hormone secretion. British Poultry Science 41:355-362. doi 10.1080/713654923

Griffin, H. D., and C. Goddard. 1994. Rapidly growing broiler (meat-type) chickens: their origin and use for comparative studies of the regulation of growth. The International journal of biochemistry 26:19-28.

Hammer, Ø., D. a. T. Harper, and P. D. Ryan. 2001. PAST: Paleontological statistics software package for education and data analysis. Palaeontologica Electronica 4:9pp.

Havenstein, G. B., P. R. Ferket, and M. A. Qureshi. 2003a. Growth, livability, and feed conversion of 1957 versus 2001 broilers when fed representative 1957 and 2001 broiler diets. Poultry Science 82:1500-1508.

Havenstein, G. B., P. R. Ferket, and M. A. Qureshi. 2003b. Carcass composition and yield of 1957 versus 2001 broilers when fed representative 1957 and 2001 broiler diets. Poultry Science 82:1509-1518.

Havenstein, G. B., P. R. Ferket, S. E. Scheideler, and B. T. Larson. 1994a. Growth, livability, and feed conversion of 1957 vs 1991 broilers when fed "typical" 1957 and 1991 broiler diets. Poultry Science 73:1785-1794.

Havenstein, G. B., P. R. Ferket, S. E. Scheideler, and D. V. Rives. 1994b. Carcass composition and yield of 1991 vs 1957 broilers when fed "typical" 1957 and 1991 broiler diets. Poultry Science 73:1795-1804.

Hogg, D. A. 1980. A re-investigation of the centres of ossification in the avian skeleton at and after hatching. Journal of Anatomy 130:725-743. 
374 Iji, P. A., A. Saki, and D. R. Tivey. 2001. Body and intestinal growth of broiler chicks on a

375 commercial starter diet. 1. Intestinal weight and mucosal development. British Poultry Science

376 42:505-513. doi 10.1080/00071660120073151

377 Jackson, S., and J. Diamond. 1996. Metabolic and digestive responses to artificial selection in 378 chickens. Evolution 50:1638-1650. doi 10.2307/2410900

379 Julian, R. J. 1993. Ascites in poultry. Avian Pathology 22:419-454. doi $380 \quad 10.1080 / 03079459308418934$

381

382

383

384

385

386

387

388

389

390

391

392

393

394

395

396

397

398

399

400

401

402

403

404

405
Julian, R. J. 1998. Rapid growth problems: ascites and skeletal deformities in broilers. Poultry Science 77:1773-1780.

Kestin, S. C., T. G. Knowles, A. E. Tinch, and N. G. Gregory. 1992. Prevalence of leg weakness in broiler chickens and its relationship with genotype. The Veterinary record 131:190-194.

Knowles, T. G., S. C. Kestin, S. M. Haslam, S. N. Brown, L. E. Green, A. Butterworth, S. J. Pope, D. Pfeiffer, and C. J. Nicol. 2008. Leg disorders in broiler chickens: prevalence, risk factors and prevention. PLoS ONE 3:e1545. doi 10.1371/journal.pone.0001545

Konarzewski, M., A. Gavin, R. Mcdevitt, and I. R. Wallis. 2000. Metabolic and organ mass responses to selection for high growth rates in the domestic chicken (Gallus domesticus). Physiological and Biochemical Zoology 73:237-248. doi 10.1086/316729

Lieber, R. L., and J. Friden. 2000. Functional and clinical significance of skeletal muscle architecture. Muscle \& nerve 23:1647-1666.

Markley, J. S., and D. R. Carrier. 2010. The cost of ventilation in birds measured via unidirectional artificial ventilation. Comparative biochemistry and physiology. Part A, Molecular \& integrative physiology 155:146-153. doi 10.1016/j.cbpa.2009.10.023

Maruyama, K., M. K. Akbar, and C. M. Turk. 1999. Growth pattern and carcase development in male ducks selected for growth rate. British Poultry Science 40:233-239.

Maxwell, E. E. 2008. Comparative embryonic development of the skeleton of the domestic turkey (Meleagris gallopavo) and other galliform birds. Zoology 111:242.

Menna, T. M., and J. P. Mortola. 2002. Metabolic control of pulmonary ventilation in the developing chick embryo. Respiratory Physiology \& Neurobiology 130:43.

Paxton, H., N. B. Anthony, S. A. Corr, and J. R. Hutchinson. 2010. The effects of selective breeding on the architectural properties of the pelvic limb in broiler chickens: a comparative study across modern and ancestral populations. Journal of Anatomy 217:153-166. doi $10.1111 / \mathrm{j} .1469-7580.2010 .01251 . \mathrm{x}$ 
406 Paxton, H., M. Daley, S. Corr, and J. Hutchinson. 2013. The gait dynamics of the modern broiler

407 chicken: A cautionary tale of selective breeding. The Journal of experimental biology 216:3237-

408 3248. doi 10.1242/jeb.080309

409 Randall, C. J. 1982. Acute pectoral myopathy in broiler breeders. Avian Pathology 11:245-252. 410 doi 10.1080/03079458208436098

411 Rayner, J. M. V. 1985. Linear relations in biomechanics: the statistics of scaling functions. 412 Journal of Zoology 206:415-439.

413 Scheid, P., and J. Piiper. 1971. Direct measurement of the pathway of respired gas in duck lungs. 414 Respiration Physiology 11:308-314.

Schmidt, C. J., M. E. Persia, E. Feierstein, B. Kingham, and W. W. Saylor. 2009. Comparison of a modern broiler line and a heritage line unselected since the 1950s. Poultry Science 88:2610-2619. doi Doi 10.3382/Ps.2009-00055

418 Schmidt-Nielsen, K. 1984. Scaling : why is animal size so important? CUP.

Sokal, R. R., and F. J. Rohlf. 1995. Biometry : the principles and practice of statistics in 420 biological research. 3rd ed. ed. Freeman, New York ; Oxford.

Swatland, H. J. 1979. Development of shape in turkey carcasses. Journal of Agricultural Science 422 93:1-6.

423 Thaxton, J. P. 2002. Heart growth in broilers. British Poultry Science 43:24-27. doi $424 \quad 10.1080 / 00071660120109827$

425 Tickle, P. G., and J. R. Codd. 2009. Ontogenetic development of the uncinate processes in the 426 domestic turkey (Meleagris gallopavo). Poultry Science 88:179-184. doi Doi 10.3382/Ps.200842700349 significance of the uncinate processes in birds. Journal of Experimental Biology 210:3955-3961.

430 doi 10.1242/jeb.008953

431 Tickle, P. G., R. L. Nudds, and J. R. Codd. 2012. Barnacle geese achieve significant energetic 432 savings by changing posture. PLoS ONE 7:e46950. doi 10.1371/journal.pone.0046950

433 Tickle, P. G., M. F. Richardson, and J. R. Codd. 2010. Load carrying during locomotion in the 434 barnacle goose (Branta leucopsis): The effect of load placement and size. Comparative 435 Biochemistry and Physiology a-Molecular \& Integrative Physiology 156:309-317. doi Doi 436 10.1016/J.Cbpa.2010.01.022 
437 Viera, S. L., and E. T. Moran, Jr. 1999. Effects of egg of origin and chick post-hatch nutrition on 438 broiler live performance and meat yields. World's Poultry Science Journal 55:126-142.

439 Wideman, R. F. 2001. Pathophysiology of heart/lung disorders: pulmonary hypertension 440 syndrome in broiler chickens. Worlds Poultry Science Journal 57:289-307. doi Doi $441 \quad 10.1079 / \mathrm{Wps} 20010021$

442 Wilson, J. B., R. J. Julian, and I. K. Barker. 1988. Lesions of right heart failure and ascites in 443 broiler chickens. Avian Diseases 32:246-261.

444 Zimmer, K. 1935. Beitrage zur Mechanik der Atmung bei den Vögeln in Stand und Flug. 445 Aufgrund anatomischer-physiologisher und experimenteller Studien. Zoologica 88:1-69. 


\section{Table 1 (on next page)}

Internal organ growth over development.

Anatomical and biomechanical traits of broiler chickens across ontogeny. 1. Anatomy of the musculoskeletal respiratory apparatus and changes in organ size. Internal organ mass as a proportion of total body mass $\left(M_{\mathrm{b}}\right)$ over development. Data are mean \pm standard deviation. Asterisk $\left(^{*}\right)$ denotes significant differences at the 0.05 level. 
Table 1

\begin{tabular}{|c|c|c|c|c|c|c|}
\hline Age (days) & $\mathbf{n}$ & $M_{\mathrm{b}}(\mathrm{kg})$ & Heart $\left(\% M_{\mathrm{b}}\right)$ & Lung $\left(\% M_{\mathrm{b}}\right)$ & $\operatorname{Liver}\left(\% M_{\mathrm{b}}\right)$ & Intestine $\left(\% M_{\mathrm{b}}\right)$ \\
\hline 1 & $\begin{array}{l}1 \\
0 \\
1\end{array}$ & $0.04 \pm 0.003 *$ & $0.74 \pm 0.05$ & $1.04 \pm 0.20^{*}$ & $2.99 \pm 0.34^{*}$ & $17.1 \pm 2.71 *$ \\
\hline 14 & $\begin{array}{l}1 \\
0\end{array}$ & 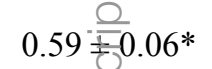 & $0.71 \pm 0.10$ & $0.75 \pm 0.16^{*}$ & $3.63 \pm 0.30$ & $9.89 \pm 1.05^{*}$ \\
\hline 28 & $\begin{array}{l}9 \\
1\end{array}$ & $1.40 \stackrel{\stackrel{ \pm 2}{\simeq} 0.09 *}{\subseteq}$ & $0.66 \pm 0.06$ & $0.72 \pm 0.09^{*}$ & $3.10 \pm 0.32$ & $8.27 \pm 0.90^{*}$ \\
\hline 42 & 3 & 3.27 원 0.21 * & $0.53 \pm 0.07 *$ & $0.55 \pm 0.12 *$ & $2.48 \pm 0.22$ & $5.34 \pm 0.51 *$ \\
\hline
\end{tabular}

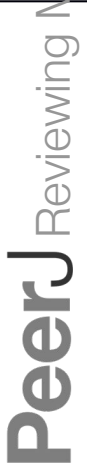




\section{Table 2 (on next page)}

Scaling relationship between organ and body mass.

The regression slope indicates the proportional change of organ mass with increasing body mass, and 95\% confidence intervals are shown (95\% CL). Isometric (=) and negative allometric (-) growth are indicated by symbols. 
Table 2

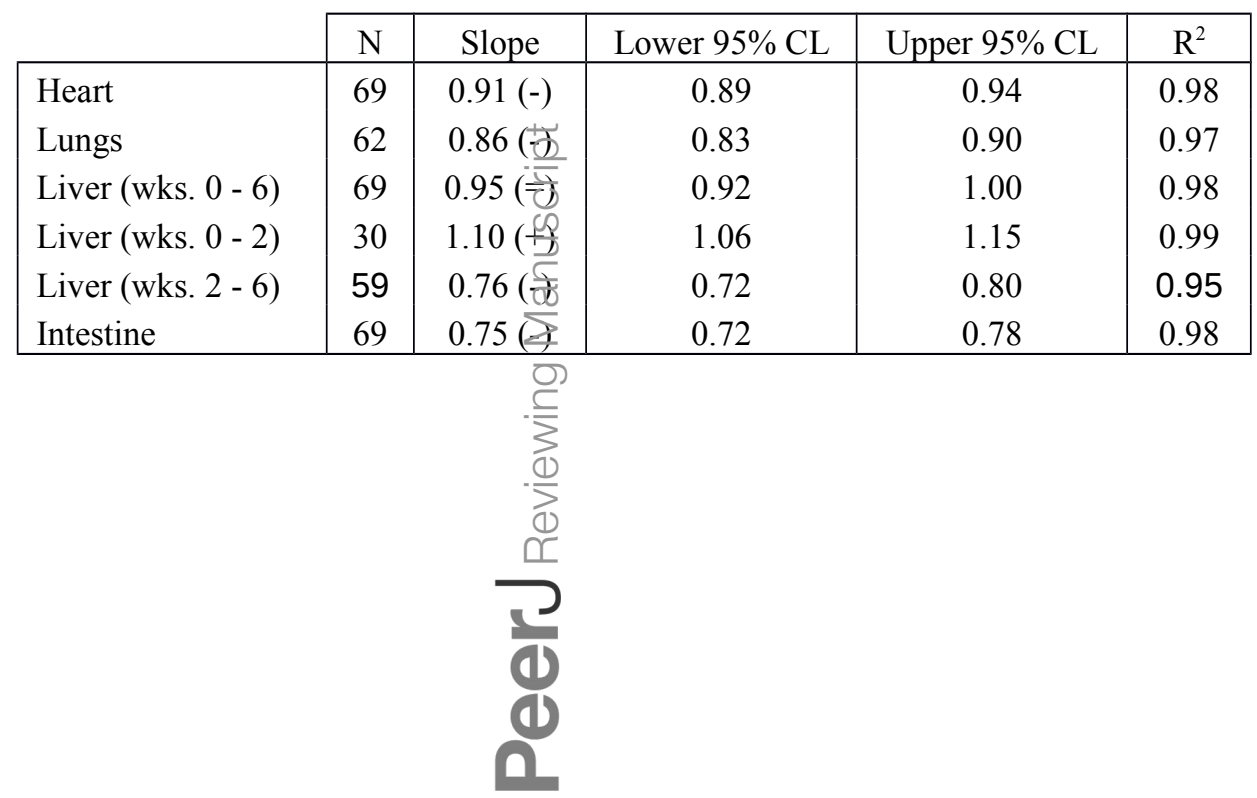




\section{Table 3 (on next page)}

Morphological examination of the external body.

Data represented are mean \pm standard deviation. Following the principles of geometric similarity (Alexander et al., 1981), girth and keel length are normalised by body mass ${ }^{1 / 3}$ to negate the effect of body size on our data. Asterisk $\left({ }^{*}\right)$ denotes significant differences at the 0.05 level. 
Table 3

\begin{tabular}{|c|c|c|c|c|c|}
\hline Age (days) & $\mathbf{n}$ & Wings $\left(\% M_{\mathrm{b}}\right)$ & Girth & Keel length & Rib Cage $\left(\% M_{b}\right)$ \\
\hline 14 & 10 & $7.5 \pm 06$ & $0.21 \pm 0.01 *$ & $0.08 \pm 0.004^{*}$ & $10.0 \pm 1.1$ \\
\hline 28 & 9 & $7.8 \pm \overline{0.5}$ & $0.23 \pm 0.01 *$ & $0.07 \pm 0.004 *$ & $7.49 \pm 0.9^{*}$ \\
\hline 42 & 12 & $5.8 \pm 6.4$ & $0.25 \pm 0.01^{*}$ & $0.10 \pm 0.005 *$ & $8.9 \pm 0.7$ \\
\hline
\end{tabular}




\section{Table 4 (on next page)}

Breast muscle growth over development.

Anatomical and biomechanical traits of broiler chickens across ontogeny. 1. Anatomy of the musculoskeletal respiratory apparatus and changes in organ size. Breast muscle mass (\% $M_{\mathrm{b}}$ ) over development. Data are mean \pm standard deviation. Asterisk $\left({ }^{*}\right)$ denotes significant differences at the 0.05 level. 
Table 4

\begin{tabular}{|c|c|c|}
\hline Age (days) & Pectoralis major $\left(\boldsymbol{\%} \boldsymbol{M}_{\mathbf{b}}\right)$ & Pectoralis minor $\left(\mathbf{\%} \boldsymbol{M}_{\mathbf{b}}\right)$ \\
\hline 1 & $0.58 \pm 0.05^{*}$ & $0.12 \pm 0.02^{*}$ \\
14 & $8.65 \pm 0.98^{*}$ & $1.88 \pm 0.27^{*}$ \\
28 & $12.12 \pm 1.11^{*}$ & $2.51 \pm 0.31^{*}$ \\
42 & $14.50 \pm 1.7 \%$ & $3.10 \pm 0.37^{*}$ \\
\hline
\end{tabular}




\section{Table 5 (on next page)}

Positive allometric growth of breast $M_{\mathrm{m}}$. 
Table 5

\begin{tabular}{|c|c|c|c|c|c|c|}
\hline & $\begin{array}{c}\text { Age } \\
\text { (weeks) }\end{array}$ & $\mathbf{N}$ & Slope & $\begin{array}{r}\text { Lower } \\
95 \% \text { CL }\end{array}$ & $\begin{array}{c}\text { Upper } \\
\text { 95\% CL }\end{array}$ & $\mathbf{R}^{2}$ \\
\hline \multirow[t]{3}{*}{$\begin{array}{l}\text { pectoralis major } \\
\text { (M. pectoralis) }\end{array}$} & $0-6 \stackrel{\varrho}{=}$ & 69 & 1.60 & 1.56 & 1.66 & 0.98 \\
\hline & $0-2$ & 30 & 1.83 & 1.77 & 1.88 & 0.99 \\
\hline & $2-6 \supseteq$ & 59 & 1.29 & 1.24 & 1.34 & 0.98 \\
\hline \multirow{3}{*}{$\begin{array}{l}\text { pectoralis minor } \\
\text { (M. supracoracoideus) }\end{array}$} & $\sum_{0}^{\infty}$ & 68 & 160 & & 170 & \\
\hline & $0-2$ & 30 & $\begin{array}{l}1.02 \\
1.87\end{array}$ & $\begin{array}{l}1.51 \\
1.79\end{array}$ & $\begin{array}{l}1.70 \\
1.95\end{array}$ & $\begin{array}{l}0.98 \\
0.98\end{array}$ \\
\hline & $2-6$ & 58 & 1.29 & 1.22 & 1.36 & 0.96 \\
\hline \multirow{2}{*}{\multicolumn{7}{|c|}{ d }} \\
\hline \multirow{2}{*}{\multicolumn{7}{|c|}{3}} \\
\hline & & & & & & \\
\hline \multicolumn{7}{|c|}{ d } \\
\hline \multicolumn{7}{|c|}{ d } \\
\hline
\end{tabular}




\section{Table 6 (on next page)}

Scaling relationships of thoracic musculoskeletal parameters and body mass.

The regression slope indicates proportional change with increasing body mass. Isometric (=), positive (+) and negative (-) allometric growth are indicated by symbols. 


\section{Table 6}

\begin{tabular}{|c|c|c|c|c|c|c|c|}
\hline & & & $\mathbf{n}$ & Slope & Lower $95 \%$ CL & Upper $95 \%$ CL & $\mathbf{R}^{2}$ \\
\hline \multirow[t]{3}{*}{ pectoralis major } & $M_{\mathrm{m}}$ & 은 & 69 & $1.60(+)$ & 1.56 & 1.66 & 0.98 \\
\hline & & @ & 37 & $0.46(+)$ & 0.42 & 0.50 & 0.95 \\
\hline & PCSA & $\vec{\partial}$ & 37 & $1.23(+)$ & 1.19 & 1.27 & 0.99 \\
\hline \multirow[t]{3}{*}{ pectoralis minor } & $M_{\mathrm{m}}$ & $\widetilde{\sigma}$ & 68 & $1.62(+)$ & 1.57 & 1.70 & 0.98 \\
\hline & & & 34 & $0.55(+)$ & 0.50 & 0.62 & 0.89 \\
\hline & PCSA & & 29 & $1.17(+)$ & 1.09 & 1.25 & 0.97 \\
\hline \multirow[t]{3}{*}{ external oblique } & $M_{\mathrm{m}}$ & s & 25 & $0.97(=)$ & 0.84 & 1.09 & 0.89 \\
\hline & & (1) & 15 & $0.31(=)$ & 0.12 & 0.48 & 0.32 \\
\hline & PCSA & $\simeq$ & 15 & $0.90(=)$ & 0.72 & 1.09 & 0.86 \\
\hline \multirow[t]{2}{*}{ sternal keel } & length & 3 & 34 & $0.48(+)$ & 0.44 & 0.51 & 0.97 \\
\hline & depth & & 34 & $0.55(+)$ & 0.51 & 0.59 & 0.95 \\
\hline uncinate process & length & & 31 & $0.30(-)$ & 0.28 & 0.32 & 0.94 \\
\hline
\end{tabular}




\section{Table 7 (on next page)}

Ossification of the uncinate processes.

Anatomical and biomechanical traits of broiler chickens across ontogeny. 1. Anatomy of the musculoskeletal respiratory apparatus and changes in organ size. Structural changes in uncinate process 4 over development from embryo ( 6 days before hatch) to slaughter age; presence of cartilage and bone as derived from stained specimens. Data presented as mean \pm standard deviation (SD). 
Table 7

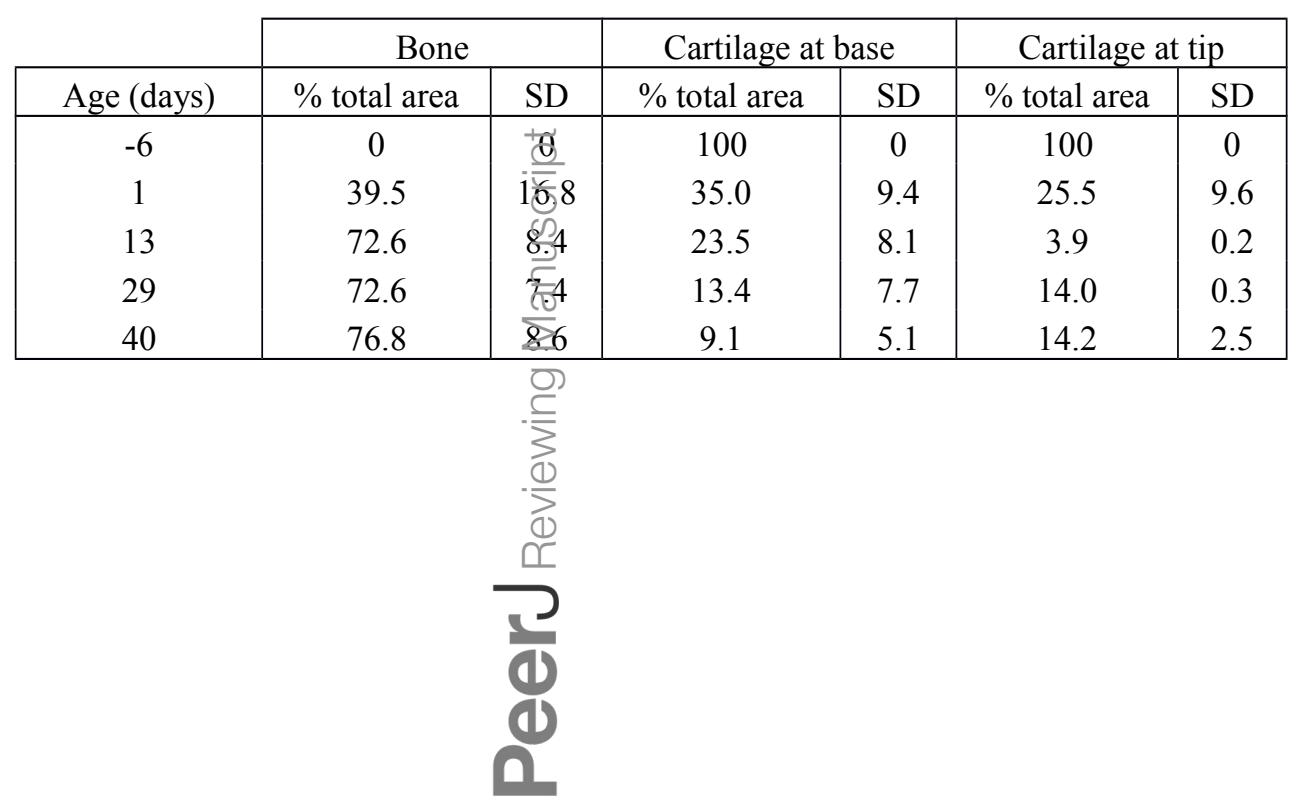




\section{Figure 1}

Breast muscle growth during development

Scatter plots showing log transformed pectoralis major and minor masses against log body mass over development from 0 - 2-weeks (solid line) and 2 - 6-weeks old (dashed line). The equations describing lines of best fit were: $(\mathrm{A})$ pectoralis major $0-2$ weeks: $M_{\mathrm{b}}{ }^{1.83}-3.28(n$ $\left.=30, r^{2}=0.99 ; \mathrm{p}<0.001\right) ; 2-6$ weeks: $M_{b}{ }^{1.29}-1.83\left(n=59 ; r^{2}=0.98 ; p<0.001\right)$. (B) pectorals minor 0 - 2 weeks: $M_{b}{ }^{1.87}-4.06\left(n=30, r^{2}=0.98 ; p<0.001\right) ; 2-6$ weeks: $M_{b}{ }^{1.28}$ $-2.48\left(n=58 ; r^{2}=0.96 ; p<0.001\right)$. 

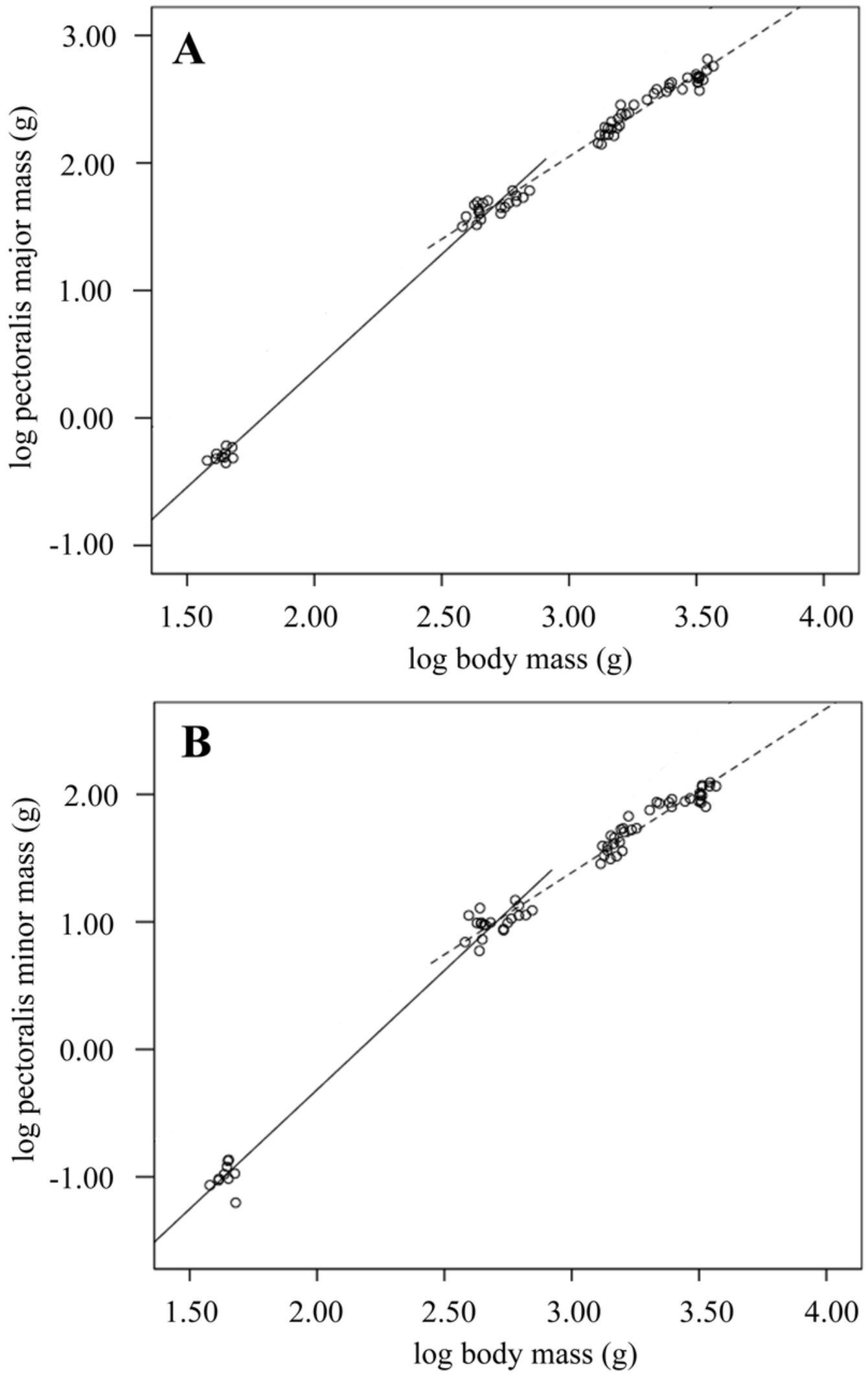


\section{Figure 2}

Ossification of the thoracic skeleton

Representative stained skeletons showing the progression of ossification in the vertebral ribs, uncinate processes and sternum. Blue areas correspond to cartilage and red areas to bone. Ossification of ribs and uncinate processes are shown for the hatchling ( $A$ and $B)$, 2-week old $(C$ and $D)$ and 6-week old ( $E$ and $F$ ) birds panels. Ossification of the uncinate processes and sternum remain incomplete at slaughter age. Scale bars represent $10 \mathrm{~mm}$. 


\section{Figure 3}

Organ growth during development

Scatter plots showing log transformed organ masses against log body mass over development from hatch to 6-weeks old. The equations describing lines of best fit were: $(A)$ heart: $M_{\mathrm{b}}{ }^{0.91}-1.96 ;\left(n=69 ; r^{2}=0.98 ; \mathrm{p}<0.001\right)$; (B) lung: $M_{\mathrm{b}}{ }^{0.86}-1.76\left(n=62 ; r^{2}=0.97\right.$; $\mathrm{p}<0.001) ;(\mathrm{C})$ liver: $0-2$ weeks old (solid line): $M_{\mathrm{b}}{ }^{1.10}-1.70\left(n=30 ; r^{2}=0.99 ; p<0.001\right)$; $2-6$ weeks old (dashed line): $M_{\mathrm{b}} 0.76-0.77\left(n=59 ; r^{2}=0.95 ; \mathrm{p}<0.001\right)$; (D) intestine: $M_{\mathrm{b}} 0.75-0.34\left(n=69 ; r^{2}=0.98 ; \mathrm{p}<0.001\right)$.
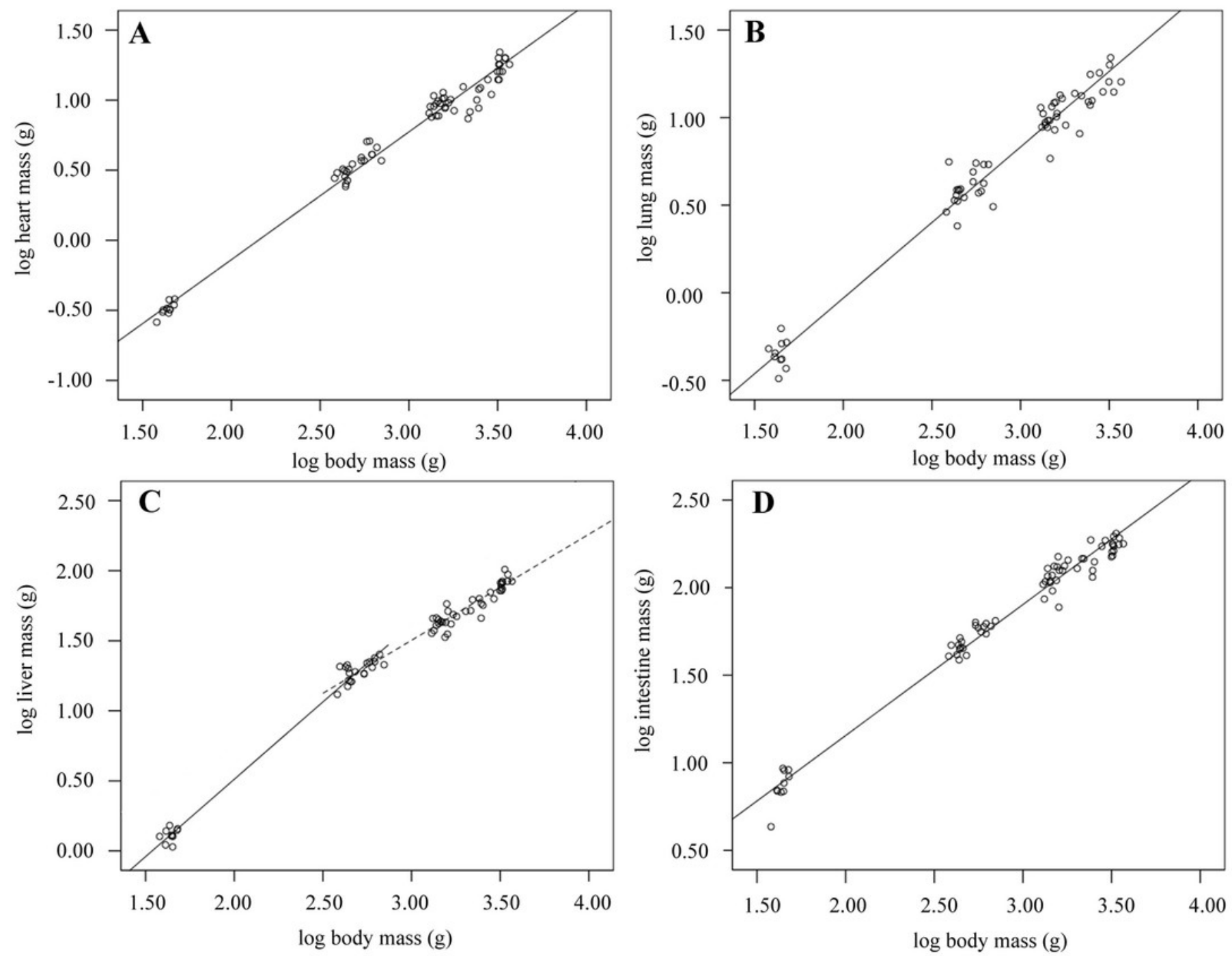